\title{
Broad line emission in dwarf galaxies: the first detection of low-metallicity AGN
}

\author{
Yuri I. Izotov \\ Main Astronomical Observatory, \\ 27 Zabolotnoho str., Kyiv, 03680, Ukraine \\ email: izotov@mao.kiev.ua
}

\begin{abstract}
Observations of AGN show that they generally possess a high metallicity, varying from solar to supersolar metallicities. This is the case since AGN are usually found in massive, bulge-dominated galaxies that have converted most of their gas into stars by the present epoch. Since AGN metallicity is strongly correlated with stellar mass, low-metallicity AGN are expected to be in low-mass dwarf galaxies. However, until now, searches in low-mass galaxies have only turned up AGN with metallicities around half that of typical AGN, i.e. with solar or slightly subsolar values. We report the discovery of four low-metallicity dwarf galaxies in the Data Release 6 of the Sloan Digital Sky survey, with $12+\log \mathrm{O} / \mathrm{H}$ in the range 7.4-8.0, and that appear to harbor an AGN. In the course of a long-range program to search for extremely metaldeficient emission-line dwarf galaxies, we have come across four galaxies with very unusual spectra: the strong permitted emission lines, mainly the $\mathrm{H} \alpha$ line, show very prominent broad components, with full widths at zero intensity corresponding to velocities varying between 2200 and $3500 \mathrm{~km} \mathrm{~s}^{-1}$, and extraordinarily large broad $\mathrm{H} \alpha$ luminosities, varying from $3 \times 10^{41}$ to $2 \times 10^{42} \mathrm{erg} \mathrm{s}^{-1}$. The Balmer lines show a very steep decrement, suggesting collisional excitation and that the broad emission comes from very dense gas $\left(N_{e} \gg 10^{4} \mathrm{~cm}^{-3}\right)$. Only the presence of an accretion disk around an intermediate-mass black hole in the dwarf galaxies appears to account for these properties.
\end{abstract}

Keywords. galaxies: active, galaxies: abundances, galaxies: dwarf, galaxies: nuclei

\section{Introduction}

Active galactic nuclei (AGN) are usually found in massive, bulge-dominated galaxies that have converted most of their gas into stars by the present epoch, therefore their gas metallicities are generally high (Storchi-Bergmann et al. 1998, Hamann et al. 2002). A question then arises: do low-metallicity AGN exist? If so, can we find them in low-mass galaxies? To address these questions, Groves et al. (2006) have searched the Sloan Digital Sky Survey (SDSS) Data Release 4 (DR4) spectroscopic galaxy sample. Using diagnostic line ratios and imposing an upper mass limit of $10^{10} M_{\odot}$ to restrict themselves to lowmass galaxies, they are left with a sample of only $\sim 40$ AGN, which they found to appear to have metallicities around half that of typical AGN, i.e. solar or slightly subsolar values. The same high metallicity range is found in the sample of low-mass AGN of Greene \& Ho (2007). Assessing their findings, Groves et al. (2006) are led to another question: "Why are there no AGN with even lower metallicities?" Here, following Izotov \& Thuan (2008), we suggest that these low-metallicity AGN do exist although they are extremely rare.

In the course of a long-range program to search for extremely metal-deficient emissionline dwarf galaxies, Izotov et al. (2007) have used the SDSS DR5 database. While studying that sample to look for emission-line galaxies (ELGs) with broad components in their strong emission lines, Izotov et al. (2007) came across four galaxies with very unusual spectra. Their spectra shown in Fig. 1, resemble those of moderately to very 
low-metallicity high-excitation $\mathrm{H}$ II regions: their oxygen abundances are in the range $12+\log \mathrm{O} / \mathrm{H} \sim 7.4-8.0$. Izotov et al. (2007) found that there is however a striking difference: the strong permitted emission lines, mainly the $\mathrm{H} \alpha \lambda 6863$ line, show very prominent broad components. These are characterized by somewhat unusual properties: 1) their $\mathrm{H} \alpha$ full widths at zero intensity $F W Z I$ vary from 102 to $158 \AA$, corresponding to expansion velocities between 2200 and $3500 \mathrm{~km} \mathrm{~s}^{-1} ; 2$ ) the broad $\mathrm{H} \alpha$ luminosities $L_{b r}$ are extraordinarily large, varying from $3 \times 10^{41}$ to $2 \times 10^{42} \mathrm{erg} \mathrm{s}^{-1}$. This is to be compared with the range $10^{37}-10^{40} \mathrm{erg} \mathrm{s}^{-1}$ found by Izotov et al. (2007) for the other ELGs with broad-line emission; 3) the Balmer lines show a very steep decrement, suggesting collisional excitation and that the broad emission comes from very dense gas $\left(N_{e} \gg 10^{4} \mathrm{~cm}^{-3}\right)$. The very large $\mathrm{H} \alpha$ luminosities are most likely associated with SN shocks or AGN. Izotov et al. (2007) have considered type IIn SNe because their H $\alpha$ luminosities are larger $\left(\sim 10^{38}-10^{41}\right.$ $\operatorname{erg~s}^{-1}$ ) than those of the other SN types and they decrease less rapidly.

\section{Broad emission and diagnostic diagrams}

To decide whether type IIn SNe or AGN are responsible for the broad emission in these galaxies, monitoring of their spectral features on the relatively long time scale of several years is necessary. If broad features are produced by IIn type SNe, then we would expect a decrease in the broad line luminosities. No significant temporal evolution would be expected in the case of an AGN. In order to check for temporal evolution, Izotov \& Thuan (2008) obtained second-epoch spectra of the above four galaxies with broad emission, using the $3.5 \mathrm{~m}$ Apache Point Observatory (APO) telescope.

Comparison of SDSS and APO broad $\mathrm{H} \alpha$ fluxes shows that they have remained nearly constant (with variations $\leqslant 20 \%$ ) over a period of $\sim 3-7$ years. This likely rules out the hypothesis that the broad line fluxes are due to type IIn SN because their $\mathrm{H} \alpha$ fluxes should have decreased significantly over this time interval.

There remains the AGN scenario. Can accretion disks around black holes in these low-metallicity dwarf galaxies account for their spectral properties? The spectra of the four objects do not show clear evidence for the presence of an intense source of hard nonthermal radiation: the [Ne v] $\lambda 3426$, [O II] $\lambda 3727$, He II $\lambda 4686$, [O I] $\lambda 6300$, [N II] $\lambda 6583$, and $[\mathrm{S} \mathrm{II}] \lambda \lambda 6717,6731$ emission lines, which are usually found in the spectra of AGN, are weak or not detected. Aside from He II $\lambda 4686$, the apparent weakness of such emission lines, however, may be accounted for by the low metallicities of our galaxies. Another way to check for the presence of an AGN in a galaxy is to check for its location in the emission-line diagnostic diagram of Baldwin et al. (1981) (BPT). It can be seen in Fig. 2a that all four objects lie in the region corresponding to star-forming galaxies (SFG), to the left of the region occupied by AGN with low-mass black holes and with metallicities ranging from 2 to $1 / 4$ that of the Sun (Greene \& Ho 2007). However, their locations in the SFG region do not necessarily disqualify them as AGN candidates. Photoionization models of AGN show that lowering their metallicity moves them to the left of the BPT diagram, so that they end up in the SFG region (Groves et al. 2006, Stasińska et al. 2006). Thus the BPT diagram is unable to distinguish between SFGs and low-metallicity AGN. Admitting that there is an AGN in our dwarf galaxies, can we account for the weakness of the high-ionization lines? Photoionization models with only AGN nonthermal ionizing radiation do predict detectable He II $\lambda 4686$ and [Ne v] $\lambda 3426$ emission lines. To make the observed spectra agree with the models, one solution is to dilute the nonthermal ionizing radiation from the AGN by thermal radiation from surrounding hot massive stars. In Fig. 2a, we show the results of Izotov \& Thuan (2008) CLOUDY calculations (Ferland et al. 1998) of $\mathrm{H}$ II regions ionized by a composite radiation consisting of different proportions 
of stellar and nonthermal radiation. Two curves, characterized by different metallicities, are shown by solid lines: the lower one is for $12+\log \mathrm{O} / \mathrm{H}=7.3$ and the upper one is for $12+\log \mathrm{O} / \mathrm{H}=7.8$, typical of the metallicities of our objects. Each model point is labeled by the ratio $R$ of nonthermal-to-thermal ionizing radiation. A slope $\alpha=-1$ has been adopted for the non-thermal power-law spectrum over the whole wavelength range under consideration $\left(f_{\nu} \propto \nu^{\alpha}\right)$. The calculations have been done with a number of ionizing photons $Q_{t h}=10^{53} \mathrm{~s}^{-1}$ for stellar radiation, $Q_{n o n t h}=R Q_{t h}$ for nonthermal radiation and $N_{e}=10^{4} \mathrm{~cm}^{-3}$. Higher densities would move the curves to the right. The dotted lines in Fig. 2a show the corresponding models with $\alpha=-2$. They are very similar to the models with $\alpha=-1$ when $R \leqslant 1$, but fall below for $R \geqslant 1$. It is seen that models with $12+\log \mathrm{O} / \mathrm{H}=7.8$ and in which the nonthermal ionizing radiation contributes $\leqslant 10 \%$ of the total ionizing radiation can account well for the location of all four galaxies in the BPT diagram, independently of the slope of the power-law spectrum.

How about the high-ionization lines? In Fig. 2b, we show the diagnostic diagram for $[\mathrm{Ne}$ v] $\lambda 3426 / \mathrm{H} \beta$ vs. [N II] $\lambda 6583 / \mathrm{H} \alpha$ (thick lines) and $\mathrm{He}$ II $\lambda 4686 / \mathrm{H} \beta$ vs. [N II] $\lambda 6583 / \mathrm{H} \alpha$ (thin lines). As in Fig. 2a, CLOUDY models with $\alpha=-1$ and -2 are shown by solid and dotted lines. The vertical dashed line separates models with $12+\log \mathrm{O} / \mathrm{H}=7.3$ (Fig. $2 \mathrm{~b}$, left) from those with $12+\log \mathrm{O} / \mathrm{H}=7.8$ (Fig. $2 \mathrm{~b}$, right). The shaded rectangle shows the region of the upper limits of $\sim 1 \%-2 \%$ of the $\mathrm{H} \beta$ flux, set for $[\mathrm{Ne} \mathrm{v}] \lambda 3426 / \mathrm{H} \beta$ and $\mathrm{He}$ II $\lambda 4686 / \mathrm{H} \beta$ in our objects, in the observed range of their [N II] $\lambda 6583 / \mathrm{H} \alpha$ ratio. If we adopt $12+\log \mathrm{O} / \mathrm{H}=7.8$ as typical for our galaxies, then Fig. $2 \mathrm{~b}$ shows that models that satisfy the non-detectability limit of the high-ionization lines (i.e. that fall within the shaded box $)$ are characterized either by a steep slope and a not excessively small $R$ ( $\alpha=$ -2 and $R \sim 0.1$ ) or by a shallower slope and a very low $R(\alpha=-1$ and $R \sim 0.03)$. It is also possible that the absence of strong high-ionization lines is caused by a high covering factor of the accretion disk. In this case the hard radiation would be absorbed inside the dense accretion disk and no high-ionization forbidden lines would be formed.

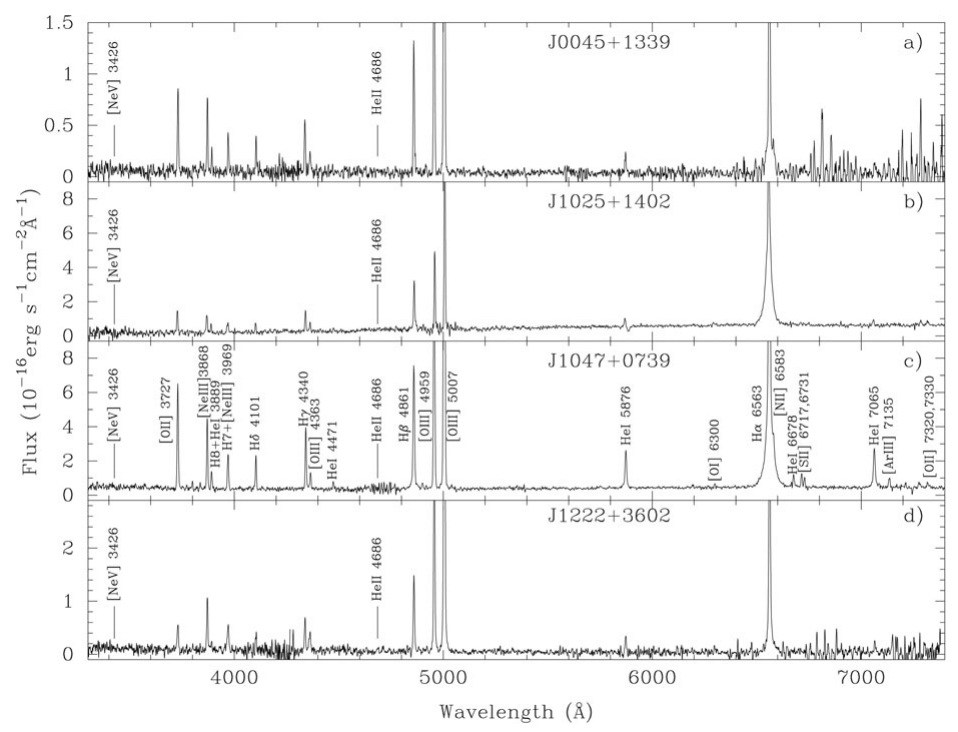

Figure 1. Redshift-corrected $3.5 \mathrm{~m}$ Apache Observatory second-epoch spectra of four low-metallicy emission-line dwarf galaxies thought to contain AGN. The locations of the non-detected $[\mathrm{Ne}$ v] $\lambda 3426$ and He II $\lambda 4686$ high-ionization emission lines are shown in all panels. Other emission lines are labeled in panel c). 


\section{Black hole virial masses}

It has been shown (see e.g. Kaspi et al. 2000) that continuum and broad line luminosities in AGN can be used to determine the size and geometry of the broad emission-line region and the mass of the central black hole. Examining a large sample of broad-line AGN, Greene \& Ho (2005) have found that the $\mathrm{H} \alpha$ luminosity scales almost linearly with the optical continuum luminosity and that a strong correlation exists between the $\mathrm{H} \alpha$ and $\mathrm{H} \beta$ line widths. On the basis of these two empirical correlations, those authors have derived the relations for the central black hole mass.

In Table 1 we list the extinction-corrected broad $\mathrm{H} \alpha$ luminosities $L(\mathrm{H} \alpha)$ and continuum luminosities $\lambda L_{\lambda}(5100)$ for the four galaxies, as derived from the SDSS spectra. The extinction coefficient was set equal to the one derived for the narrow Balmer hydrogen lines. Since the reddening due to dust extinction in dense regions may be larger than that derived from the narrow hydrogen emission lines, the derived $L(\mathrm{H} \alpha)$ should be considered as lower limits. The $L(\mathrm{H} \alpha)$ and $\lambda L_{\lambda}(5100)$ of our galaxies follow closely the correlation between $\mathrm{H} \alpha$ and continuum luminosities found by Greene \& Ho (2005). This implies that our galaxies are very likely the same type of objects as those considered by Greene \& Ho (2005). Therefore, we can use equations from Greene \& Ho (2005) for the determination of the central black hole masses. The masses $M_{\mathrm{BH}}(\mathrm{H} \alpha)$ and $M_{\mathrm{BH}}(5100)$ derived from the broad $\mathrm{H} \alpha$ and continuum luminosities are shown in Table 1 . These masses are in the range $\sim 5 \times 10^{5} M_{\odot}-3 \times 10^{6} M_{\odot}$, lower or similar to the mean black hole mass of 1.3 $\times 10^{6} M_{\odot}$ found by Greene \& Ho (2007) for their sample of low-mass black holes. Since the luminosities used to derive the masses of the central black holes are lower limits, the derived masses should also be considered as lower limits.
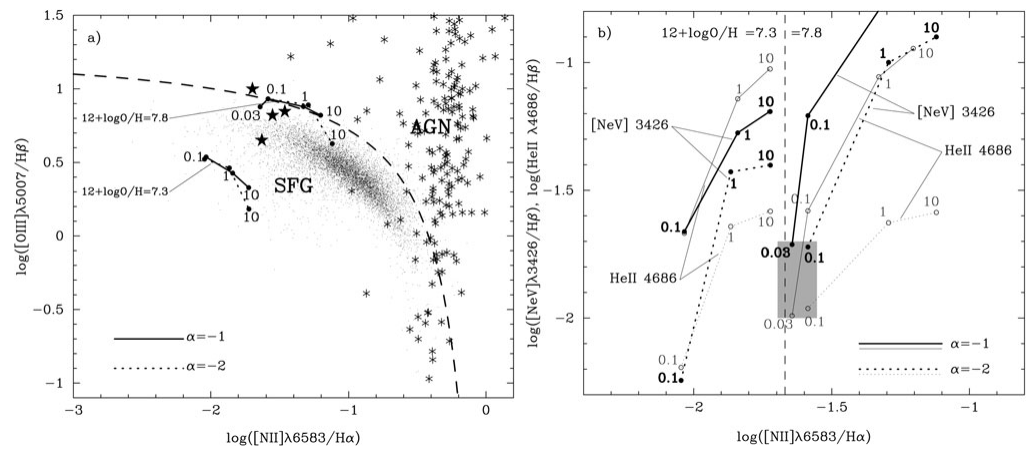

Figure 2. a) The BPT diagram (Baldwin et al. 1981) for low-ionization emission lines. Plotted are the $\sim 10,000$ ELGs from Izotov et al. (2007) (cloud of points), the low-mass black hole sample of Greene \& Ho (2007) (asterisks) and the four low-metallicity AGN in Table 1 (stars). The dashed line separates star-forming galaxies (SFG) from active galactic nuclei (AGN) (Kauffmann et al. 2003). The solid and dotted lines connect CLOUDY photoinization models computed for $\mathrm{H}$ II regions ionized by a composite radiation consisting of different proportions of stellar and nonthermal radiation. The two upper curves are characterized by $12+\log \mathrm{O} / \mathrm{H}=7.8$ and the two lower ones by $12+\log \mathrm{O} / \mathrm{H}=7.3$. Each model point is labeled by the ratio of nonthermalto-thermal ionizing radiation. All curves have been calculated adopting a number of ionizing photons $Q=10^{53} \mathrm{~s}^{-1}$ for stellar radiation, different slopes of the nonthermal spectral energy distributions $f_{\nu} \propto \nu^{\alpha}$ (solid lines are for $\alpha=-1$ and dotted lines are for $\alpha=-2$ ). A density $N_{e}$ $=10^{4} \mathrm{~cm}^{-3}$ is adopted. Higher densities would shift the curves to the right. b) The diagnostic diagram for high-ionization emission lines: [Ne v] $\lambda 3426 / \mathrm{H} \beta$ vs. [N II] $\lambda 6583 / \mathrm{H} \alpha$ (thick lines) and $\mathrm{He}$ II $\lambda 4686 / \mathrm{H} \beta$ vs. [N II] $\lambda 6583 / \mathrm{H} \alpha$ (thin lines). The same CLOUDY models as in a) are shown. The shaded region shows the upper intensity limits of the high-ionization lines [Ne $\mathrm{v}]$ $\lambda 3426$ and He II $\lambda 4686$ relative to $\mathrm{H} \beta$ in the four galaxies considered here. The dashed vertical line separates models with $12+\log \mathrm{O} / \mathrm{H}=7.30$ (left) from those with $12+\log \mathrm{O} / \mathrm{H}=7.80$ (right). 
Table 1. $\mathrm{H} \alpha$ and continuum luminosities and masses of the black holes ${ }^{1}$

\begin{tabular}{lccrrr}
\hline Object & $L_{b r}(\mathbf{H} \alpha)^{2}$ & $\mathbf{F W ~ H M}_{b r}(\mathbf{H} \alpha)^{3}$ & $\left.\lambda L_{\lambda} \mathbf{( 5 1 0 0}\right)^{2}$ & $M_{\mathrm{B} \mathrm{H}}(\mathbf{H} \alpha)^{6}$ & $M_{\mathrm{B} \mathrm{H}}(\mathbf{5 1 0 0})^{4}$ \\
\hline $\mathrm{J} 0045+1339$ & $2.74 \times 10^{41}$ & 1540 & $7.59 \times 10^{42}$ & $2.43 \times 10^{6}$ & $2.00 \times 10^{6}$ \\
$\mathrm{~J} 1025+1402$ & $3.21 \times 10^{41}$ & 680 & $3.62 \times 10^{42}$ & $5.07 \times 10^{5}$ & $2.50 \times 10^{5}$ \\
$\mathrm{~J} 1047+0739$ & $1.57 \times 10^{42}$ & 1050 & $2.32 \times 10^{43}$ & $3.05 \times 10^{6}$ & $1.91 \times 10^{6}$ \\
$\mathrm{~J} 1222+3602$ & $2.80 \times 10^{41}$ & 790 & $7.17 \times 10^{42}$ & $6.34 \times 10^{5}$ & $5.10 \times 10^{5}$ \\
\hline
\end{tabular}

Notes:

${ }^{1}$ Parameters are derived from the $2.5 \mathrm{~m}$ SDSS spectra (Izotov et al. 2007).

${ }^{2}$ In units erg s ${ }^{-1}$

${ }^{3}$ In units $\mathrm{km} \mathrm{s}^{-1}$.

${ }^{4}$ In solar masses.

\section{Conclusion}

We study here the broad line emission in four low-metallicity star-forming dwarf galaxies with $12+\log \mathrm{O} / \mathrm{H} \sim 7.4-8.0$. Our main conclusions are following:

1. The steep Balmer decrements of the broad hydrogen lines and the very high luminosities of the broad $\mathrm{H} \alpha$ line in all four galaxies $\left(3 \times 10^{41}\right.$ to $\left.2 \times 10^{42} \mathrm{erg} \mathrm{s}^{-1}\right)$ suggest that the broad emission arises from very dense and high luminosity regions such as those associated with accretion disks around black holes. If so, these four objects would harbor a new class of AGN residing in low-metallicity dwarf galaxies, with an oxygen abundance that is considerably lower than the solar or super-solar metallicity of a typical AGN.

2. There is no obvious spectroscopic evidence for the presence of a source of a nonthermal hard ionizing radiation in all four galaxies: high-ionization emission lines such as He II $\lambda 4686$ and [Ne V] $\lambda 3426$ emission lines were not detected at the level $\leqslant 1-2$ percent of the $\mathrm{H} \beta$ flux. The predicted fluxes of the high-ionization lines are below the detectability level if the spectral energy distribution $f_{\nu} \propto \nu^{\alpha}$ of the ionizing nonthermal radiation has $\alpha \sim-1$ and the nonthermal ionizing radiation is significantly diluted by the thermal stellar ionizing radiation contributing $\leqslant 3-10$ percent of the total ionizing radiation.

3. The lower limits of the masses of the central black holes $M_{\mathrm{BH}}$ of $\sim 5 \times 10^{5} M_{\odot}-$ $3 \times 10^{6} M_{\odot}$ in our galaxies are among the lowest found thus far for AGN.

\section{References}

Baldwin, J. A., Phillips, M. M., \& Terlevich, R. 1981, PASP, 93, 5

Ferland, G. J., Korista, K. T., Verner, D. A., Ferguson, J. W., Kingdon, J. B., \& Verner, E. M. 1998, PASP, 110, 761

Greene, J. E. \& Ho, L. C. 2005, ApJ, 630, 122

Greene, J. E. \& Ho, L. C. 2007, ApJ, 670, 92

Groves, B. A., Heckman, T. M., \& Kauffmann, G. 2006, MNRAS, 371, 1559

Hamann, F. et al. 2002, ApJ, 564, 592

Izotov, Y. I. \& Thuan, T. X. 2008, ApJ, in press; preprint arXiv:0807.2029

Izotov, Y. I., Thuan, T. X., \& Guseva, N. G. 2007, ApJ, 671, 1297

Kauffmann, G., et al. 2003, MNRAS, 346, 1055

Kaspi, S., Smith, P. S., Netzer, H., Maoz, D., Jannuzi, B. T., \& Giveon, U. 2000, ApJ, 533, 631

Stasińska, G., Cid Fernandes, R., Mateus, A., Sodré, L., Jr., \& Asari, N. V. 2006, MNRAS, 371, 972

Storchi-Bergmann, T., Smitt, H. R., Calzetti, D., \& Kinney, A. L. 1998, AJ, 115, 909 